\title{
Restoring detailed balance in the Landau-Teller probabilities for collision-induced vibrational transitions
}

\author{
E. E. Nikitin ${ }^{a b}$ and J. Troe ${ }^{* a}$ \\ Received 16th December 2005, Accepted 22nd March 2006 \\ First published as an Advance Article on the web 13th April 2006 \\ DOI: $10.1039 / b 517843 f$
}

\begin{abstract}
The general quasi-classical treatment for collision-induced vibrational transitions in diatomic molecules, under near-adiabatic conditions, is used to derive quantum corrections for probabilities, calculated in the external field approximation originally used by Landau and Teller. The quantum corrections are expressed through the Landau-Teller classical collision time. The first-order correction to the classical exponent restores detailed balance for up- and downtransitions and does not depend on the properties of the bath except for its temperature. The limits of applicability of the first-order correction are discussed.
\end{abstract}

\section{Introduction}

The Landau-Teller (LT) theory of vibrational relaxation of diatomic molecules in a heat bath ${ }^{1}$ employs an external field approximation for the calculation of transition probabilities. An explicit expression for vibrational state-to-state transition probabilities was derived assuming an exponential, repulsive interaction between the colliding particles in collinear configuration. As a result, a linear dependence of the logarithm of the mean transition probability for deactivation of the first vibration state, $\left\langle P_{10}\right\rangle$, on $T^{-1 / 3}$, the famous LT plot was predicted,

$$
\ln \left\langle P_{10}\right\rangle=\text { const }-3\left(\pi^{2} \omega^{2} \mu a^{2} / 2 k T\right)^{1 / 3} .
$$

Here the values of two parameters are known (the vibrational frequency $\omega$ and the reduced mass of the collision partners $\mu$ ), while the third parameter, $a$, from an expression for the interaction potential in the form $U(R) \propto \exp (-R / a)$, can be roughly estimated within in the framework of the so-called asymptotic approach. ${ }^{2}$ For real collisions in three dimensions one also has to take into account the contribution of rotational motion to the vibrationally inelastic collision dynamics. However, for molecules that are not too light (such as $\mathrm{N}_{2}, \mathrm{CO}$ ) and with small reduced mass of the partners (such as collisions $\mathrm{N}_{2}$, $\mathrm{CO}+\mathrm{He}$ ), the properly defined collision time $\tau$ is much shorter than the rotational period $t_{\text {rot }}$ and, for molecules of low asymmetry, steep repulsion, and shallow potential wells, one can simulate the collision by a collinear event occurring at a fixed value of the angle between the collisional and the molecular axes; subsequently averaging over all angles is performed (the so-called infinite order sudden approximation, IOS, such as that demonstrated for CO-He collisions in ref. 3). One then expects that eqn (1) is applicable, with all influences of the angular dependence of the interaction absorbed into the constant term of eqn (1). As long as the condition $\tau / t_{\text {rot }} \ll 1$ is satisfied, there remains mainly two effects that can lead to

\footnotetext{
${ }^{a}$ Max-Planck-Institut für biophysikalische Chemie, Am Fassberg 11, D-37077 Goettingen, Germany

${ }^{b}$ Department of Chemistry, Technion - Israel Institute of Technology, 32000 Haifa, Israel
}

curvatures of the LT plot: deviations of the interaction potential from an exponential repulsion form and quantum corrections to the external field approximation. The former was repeatedly addressed in the literature (see, e.g. ref. 4-6 and earlier work cited therein). The latter attracted attention mainly in connection with the recovery of the quantum correlation functions of the bath from its classical counterpart (see review 7 and ref. 8-15). In particular, ref. 14 provides a list of different quantum factors that correct the classical correlation function of external force and which were derived in the various approaches. Of course, the quantum corrections for any particular collision can be easily deduced from an accurate solution of the scattering equation. However, in this way it is difficult to get a general picture. Therefore, in the present work we address this problem again, adopting the LT model of collinear collisions and resorting to the general formula for quasi-classical matrix elements in the near-adiabatic limit ${ }^{16}$ where the main dependence on the characteristic parameters of the problem comes from the exponential factor in the transition probability. This allows one to limit the discussion to the exponential factors of the transition probabilities.

\section{The Landau-Teller model}

Landau and Teller considered the collinear collision of a structureless particle A with a harmonic oscillator under the assumption that the vibrational amplitude of $\mathrm{BC}$ is small compared to the range of the intermolecular forces between $\mathrm{A}$ and $\mathrm{BC}$, and that the collision time is large compared to the period of the molecular oscillation. Explicit expressions for probabilities and rate coefficients were obtained for the case when $\mathrm{A}$ and $\mathrm{B}$ interact through an exponential repulsive potential. In a first order perturbation approach, the probability of a transition $E_{i} \rightarrow E_{f}$ between the states of the translational energies $E_{i}$ and $E_{f}$ is proportional to the square of the absolute value of the matrix element between states $E_{i}$ and $E_{f}$. In the so-called common trajectory (CT) approximation (also called the external field approximation) used by Landau and Teller, the matrix element is proportional to the Fourier component of the time-dependent perturbation. 
Landau and Teller outlined general arguments why the CT transition probability for nearly adiabatic conditions will exponentially depend on the transition frequency $\omega=|\Delta E| / \hbar$, i.e. will have the form

$$
P^{\mathrm{CT}}(\omega, E) \propto \exp (-2 \omega \tau(E))
$$

Here, $E$ is the "collision energy", and the transition frequency $\omega$, for single-quantum transitions, coincides with the frequency of the oscillator. LT also indicated how the time $\tau(E)$ could be calculated for an unperturbed trajectory of energy $E$. The exponential dependence of $P^{\mathrm{CT}}$ on the frequency $\omega$ of a quantum oscillator corresponds to a similar dependence for the change of the vibrational energy of a classical oscillator. In the latter case, the near adiabatic condition $\omega \tau \gg 1$ ensures that the change in the vibrational energy is small and, therefore, an influence of the oscillatory mode on the relative motion can be neglected. This allows one to identify the "collision energy" $E$ with the initial translational energy $E_{i}$. For a quantum oscillator, the adiabatic condition $\omega \tau \gg 1$ guarantees a small change in the mean vibrational energy, though the energy transfer $\Delta E=\hbar \omega$ may not be small. Then the "collision energy" $E$ can be affected by $\Delta E$, though in an unknown way. We note in passing that the exponential dependence of the transition probability on the transferred energy $\hbar \omega$ was later dubbed the "energy gap law".

Accepting the approximation $E=E_{i}$ as is done by LT, we write the expression for thermally averaged transition probability as

$$
\left\langle P^{\mathrm{CT}}(\Delta E)\right\rangle \propto \int \exp \left[-2 \omega \tau\left(E_{i}\right)-E_{i} / k T\right] \frac{\mathrm{d} E_{i}}{k T}
$$

LT remarked that, for $\omega \tau \gg 1$, the thermal averaging can be carried out in the steepest descent (SD) approximation. In this approximation, eqn (3) leads to

$$
\left\langle P^{\mathrm{CTSD}}(\Delta E)\right\rangle \propto \exp \left[-2 \omega \tau\left(E^{*}\right)-E^{*} / k T\right]
$$

where the $\mathrm{SD}$ energy $E^{*}$ is found from the equation

$$
-\left.2 \omega \frac{\mathrm{d} \tau}{\mathrm{d} E_{i}}\right|_{E_{i}=E^{*}}-\frac{1}{k T}=0
$$

For an exponential repulsion, one has $\tau(E)=\pi a / \sqrt{2 E / \mu}$. Substitution of this $\tau(E)$ into eqn (5) and (4), as is well known, yields the second term at the r.h.s. of eqn (1).

Since $\left\langle P^{\mathrm{CTSD}}(\Delta E)\right\rangle$ does not depend on the sign of $\Delta E$ (note that $\omega=|\Delta E| / \hbar)$, the average probabilities of up- $(\Delta E>0)$ and down- $(\Delta E<0)$ transitions are the same, i.e. $\left\langle P^{\mathrm{CTSD}}(\Delta E)\right\rangle$ $=\left\langle P^{\mathrm{CTSD}}(-\Delta E)\right\rangle$. This means that the average transition probabilities do not satisfy the detailed balance relation

$$
\left\langle P_{\text {up }}\right\rangle /\left\langle P_{\text {down }}\right\rangle=\exp (-|\Delta E| / k T)
$$

LT corrected the shortcoming of the EFSD approximation by assuming

$$
\begin{aligned}
& \left\langle P_{\text {down }}^{\mathrm{LT}}\right\rangle=\left\langle P^{\mathrm{CTSD}}(\Delta E)\right\rangle, \text { for } \Delta E<0 \\
& \left\langle P_{\mathrm{up}}^{\mathrm{LT}}\right\rangle=\exp (-\Delta E / k T)\left\langle P^{\mathrm{CTSD}}(\Delta E)\right\rangle \text {, for } \Delta E>0
\end{aligned}
$$

These relations were used to formulate relaxation equations for the population of oscillator levels ${ }^{1}$ which, much later, were solved for various initial distributions. ${ }^{17}$

\section{Quasi-classical approximation in the near-adiabatic limit}

The way of correcting the CT approximation by eqn (7) appears questionable and a more consistent procedure can be formulated if the CT approximation is derived from the quasi-classical (QC) approximation formulated by Landau ${ }^{16}$ and described in the textbook ${ }^{18}$ by Landau and Lifshitz. According to this method, which is based on the analytical continuation of the classical action integrals into the classically forbidden region of the coordinate and requires the exponentially small magnitude of the transition matrix element, the probability of the transition $E_{i} \rightarrow E_{f}$ reads

$$
P^{\mathrm{QC}}\left(E_{i}, E_{f}\right) \propto \exp \left\{-(2 / \hbar)\left|\operatorname{Re}\left(\tilde{S}_{E_{i}}-\tilde{S}_{E_{f}}\right)\right|\right\}
$$

Here, $\tilde{S}_{E_{i}}\left(\right.$ or $\left.\tilde{S}_{E_{f}}\right)$ is the classical action integral taken from the turning point for the motion across the unperturbed potential at the energy $E_{i}$ (or $E_{f}$ ) into the classically forbidden region of this potential down to a transition distance determined by the stationary phase condition for the action difference. Though an excursion into the classically forbidden region of the potential looks like a completely non-classical event, it is not quite so for potentials that are represented by analytical functions, since the full potential can be recovered from a small portion of it and, therefore, the potential in the classically forbidden region is completely defined by the potential in the classically allowed region. Therefore, one hopes to find a relation between the classical exponent in eqn (2) and the QC exponent in eqn (8). Indeed, expression (8) can be recast in the form $^{19}$

$$
P^{\mathrm{QC}}\left(E_{i}, E_{f}\right) \propto \exp \left(-(2 / \hbar)\left|\int_{E_{i}}^{E_{f}} \tau(E) \mathrm{d} E\right|\right)
$$

Since the exponent in eqn (9) is completely determined by the classical time $\tau(E)$ we dwell on the calculation of this quantity. According to LT, the time $\tau(E)$ is defined through the integral

$$
\begin{aligned}
\tau(E) & =\left|\operatorname{Im}\left\{\int_{R_{t}}^{R_{s}} \frac{\mathrm{d} R}{\sqrt{(2 / \mu)(E-U(R))}}\right\}\right| \\
& =-\int_{R_{t}}^{R_{s}} \frac{\mathrm{d} R}{\sqrt{(2 / \mu)(U(R)-E)}}
\end{aligned}
$$

Here $U(R)$ is the unperturbed interaction potential between $\mathrm{A}$ and the centre of mass of $\mathrm{BC}, R_{t}$ is the turning point for the motion in the field of the potential $U(R)$, and $R_{s}$ is the distance $R$ at which the integral can be considered as converged.

The following remarks about eqn (10) have to be made:

(a) The integration range in eqn (10) corresponds to the classically forbidden region where $U(R)>E$. Of course, the Fourier component of the perturbing force is a completely classical quantity, and it can be calculated without excursion into the classically forbidden region. In this sense, eqn (10) represents an alternative way of calculating $\tau$, being valid for any analytic function $U(R)$.

(b) The distance $R_{S}$ corresponds to quite high values of the interaction energy, $\left|U\left(R_{S}\right)\right| \gg E$. As follows from eqn (10), 
$\tau(E)$ can be interpreted as the time of mutual approach of A and $\mathrm{BC}$ in the field of an inverted potential $-U(R)$, with a negative energy $-E$ and starting from the distance $R=R_{t}$. The final distance of approach, $R=R_{s}$, is immaterial since the system here spends progressively less time, moving very fast in the region of the large negative potential $-U(R)$.

(c) Though, originally, the time $\tau(E)$ was defined for asymptotically free relative motion of $\mathrm{A}$ and $\mathrm{BC}$, eqn (10) permits a generalisation to the bound motion in the complex A-BC. This implies that eqn (9) can be applied not only to free-free transitions (vibrational-to-translational energy transfer, both $E_{i}$ and $E_{f}$ positive), but also to bound-free transitions (vibrational predissociation, $E_{i}$ negative and $E_{f}$ positive) and bound-bound transitions (intramolecular energy redistribution, $E_{i}$ and $E_{f}$ negative).

Returning to free-free transitions, we can check the accuracy of the QC approximation by comparing the QC transition probability $P^{\mathrm{QC}}$ with its accurate quantum counterpart, $P^{\mathrm{Q}}$. As an example, we take an exponential potential $U(R) \propto$ $\exp (-R / a)$ for which $P^{\mathrm{Q}}$ was calculated analytically by Jackson and Mott. ${ }^{20}$ The energy-dependent part of $P^{\mathrm{Q}}$ reads

$$
P^{\mathrm{Q}}\left(E_{i}, E_{f}\right) \propto \frac{\sinh \left(2 \pi a p_{i} / \hbar\right) \cdot \sinh \left(2 \pi a p_{f} / \hbar\right)}{\left(\cosh \left(2 \pi a p_{i} / \hbar\right)-\cosh \left(2 \pi a p_{f} / \hbar\right)\right)^{2}}
$$

where $p_{i, f}=\sqrt{2 \mu E_{i, f}}$ are the initial and final momenta associated with their respective energies. On the other hand, expression (9) with $\tau(E)=\pi a / \sqrt{2 E / \mu}$ yields

$$
P^{\mathrm{QC}}\left(E_{i}, E_{f}\right) \propto \exp \left(-\left|2 \pi a p_{i} / \hbar-2 \pi a p_{f} / \hbar\right|\right)
$$

It can be easily verified that the quantum expression of eqn (11) reduces to the QC expression of eqn (12) under the conditions

$$
2 \pi a p_{i} / \hbar \gg 1,2 \pi a p_{f} / \hbar \gg 1
$$

and

$$
\left|2 \pi a p_{i} / \hbar-2 \pi a p_{f}\right| \hbar \mid \gg 1
$$

The inequalities of eqn (13) are the standard WKB criteria for QC motion in the field of the potential $U(R) \propto \exp (-R / a)$, while eqn (14) ensures exponentially small values of the transition probability. The latter is equivalent to near-adiabatic conditions for collisions. This example explains the meaning of "quasi-classical" and "exponentially small" attributes of the Landau probabilities in the expression in eqn (8).

Considering now the average transition probability, we write it in the form

$$
\left\langle P^{\mathrm{QC}}(\Delta E)\right\rangle \propto \int_{0}^{\infty} \exp \left(-(2 / \hbar)\left|\int_{E_{i}}^{E_{i}+\Delta E} \tau(E) \mathrm{d} E\right|-\frac{E_{i}}{k T}\right) \frac{\mathrm{d} E_{i}}{k T}
$$

After using the SD approximation, eqn (15) simplifies into

$$
\left\langle P^{\mathrm{QC}}(\Delta E)\right\rangle \propto \exp \left(-(2 / \hbar)\left|\int_{E^{*}}^{E^{*}+\Delta E} \tau(E) \mathrm{d} E\right|-\frac{E^{*}}{k T}\right)
$$

where the $\mathrm{SD}$ energy $E^{*}$ is determined from the equation

$$
\left|\tau\left(E^{*}+\Delta E\right)-\tau\left(E^{*}\right)\right|-\hbar / 2 k T=0
$$

The SD energy $E^{*}$ obtained from this equation depends on the sign of $\Delta E$, but this dependence is such that the first term in the exponent of eqn (16) is independent from the sign of $\Delta E$. The SD energies for up- and down-transitions, the second term, are related by $E_{\text {up }}^{*}=E_{\text {down }}^{*}+|\Delta E|$. As a result, $\left\langle P_{\text {up }}^{\mathrm{QC}}\right\rangle$ and $\left\langle P_{\text {down }}^{\mathrm{QC}}\right\rangle$ now satisfy the detailed balance relation of eqn (6).

If the expression of the QC exponent is written as

$$
\frac{1}{\hbar}\left|\int_{E_{i}}^{E_{f}} \tau(E) \mathrm{d} E\right|=\omega \tau(\bar{E})
$$

one has a connection to the CT approximation of eqn (2). However, this is not very helpful since the quantity $\bar{E}$ depends on $E_{i}$ and $E_{f}$ in an unknown way.

A useful approximation can be obtained for small energy transfer where the integrand $\tau(E)$ can be approximated by its linear expansion

$$
\tau(E)=\tau\left(E_{i}\right)+\frac{\mathrm{d} \tau\left(E_{i}\right)}{\mathrm{d} E_{i}}\left(E-E_{i}\right)
$$

With this approximation we get

$$
\begin{aligned}
\frac{2}{\hbar}\left|\int_{E^{*}}^{E^{*}+\Delta E} \tau(E) \mathrm{d} E\right|= & 2 \frac{|\Delta E|}{\hbar} \tau\left(E_{i}\right) \\
& -\operatorname{sign}(\Delta E) \frac{(\Delta E)^{2}}{\hbar} \frac{\mathrm{d} \tau\left(E_{i}\right)}{\mathrm{d} E_{i}}
\end{aligned}
$$

Substitution of this expression into eqn (15) yields

$$
\left.\left\langle P^{\mathrm{QC}}(\Delta E)\right\rangle\right|_{\text {small }|\Delta E|} \propto \int \exp \left(-2 \omega \tau\left(E_{i}\right)-\frac{E_{i}}{k T}-\omega \Delta E \frac{\mathrm{d} \tau\left(E_{i}\right)}{\mathrm{d} E_{i}}\right) \times \frac{\mathrm{d} E_{i}}{k T}
$$

where the last term in the exponent is assumed to be small compared to the other two terms. Then the SD energy is determined by the first two terms only, i.e. as given by eqn (5). In this way, we finally obtain

$$
\left.\left\langle P^{\mathrm{QCSD}}(\Delta E)\right\rangle\right|_{\text {small }|\Delta E|}=\exp (-\Delta E / 2 k T)\left\langle P^{\mathrm{CTSD}}(\Delta E)\right\rangle
$$

where $\Delta E>0$ for up-transitions and $\Delta E<0$ for downtransitions. The correction factor in eqn (18) coincides with that suggested by Nikitin in $1959^{21}$ from symmetry considerations, by Schofield in $1960^{22}$ on the basis of an analytical continuation of the classical correlation function, and by Miklave in $1983^{23}$ who derived it from the quantum solution by Jackson and $\operatorname{Mott}^{20}$ for a model with exponential interaction. It differs, however, from other quantum correction factors derived by other authors, such as those listed in Table 1 of ref. 14. The differences are due to different conditions under which the various corrections are valid and we will not discuss this in this article. Instead we only consider the conditions of validity of the correction factor of eqn (22) which are weak coupling of the oscillator to the bath, near-adiabatic energy transfer, and values of the ratio $|\Delta E| / k T$ that are not too high. The implication of the latter condition is discussed in the next section. 


\section{The validity of the first QC correction to the CT transition probability and the generalized Landau-Teller relation}

The correction factor of eqn (22) does not depend on the properties of the heat bath except for its temperature. At the same time, this factor represents the first order quantum correction to the classical correlation function of the external force acting on the oscillator. There exist higher-order corrections and their influence on the transition probabilities can be estimated by elaborating the expansion parameters of the truncated series of eqn (19). Assuming a relation $|\mathrm{d} \tau / \mathrm{d} E| \approx$ $\tau / E$ for a smooth function $\tau(E)$, we infer that the expansion parameter is equal to $|\Delta E| / E^{*}$, while, from the SD approach, we get $E^{*} / k T=\omega \tau\left(E^{*}\right)$. Since $\omega \tau\left(E^{*}\right) \gg 1$, we have $E^{*} \gg k T$. We can therefore write the following qualitative expansion of the integral in eqn (16)

$$
\begin{aligned}
& \frac{2}{\hbar}\left|\int_{E^{*}}^{E^{*}+\Delta E} \tau(E) \mathrm{d} E\right|=2 \omega \tau\left(E^{*}\right)\left\{1+O\left(\frac{\Delta E}{E^{*}}\right)+O\left(\left(\frac{\Delta E}{E^{*}}\right)^{2}+\ldots\right)\right\} \\
& =2 \omega \tau\left(E^{*}\right) \pm \frac{|\Delta E|}{2 k T}+O\left(\left(\frac{|\Delta E|}{2 k T}\right)^{2} \frac{1}{2 \omega \tau\left(E^{*}\right)}\right)+\ldots
\end{aligned}
$$

where $O(x)$ means "of the order of $x$ ". It follows from eqn (23) that higher-order corrections produce a small relative change in the exponent of eqn (9) under the condition $|\Delta E| / 2 k T \ll$ $2 \omega \tau\left(E^{*}\right)$, while a small relative change in the exponent is ensured under the different condition

$$
(\Delta E / 2 k T)^{2} \ll 2 \omega \tau\left(E^{*}\right)
$$

Considering $\omega \tau\left(E^{*}\right) \gg 1$, the condition of eqn (24) also allows for the ratio $\hbar \omega / k T$ to be larger than unity. This means that eqn (21) also includes the range of quantum vibrations where $\hbar \omega / k T \geq 1$ is fulfilled. Note, however, that the allowed range of the ratio $\hbar \omega / k T$ is limited from above by the condition of eqn (24).

The generalized LT relation now can be rewritten as

$$
\begin{aligned}
\ln \left\langle P_{10}\right\rangle= & \text { const }-3 \Xi(\omega, T)+\frac{\hbar \omega}{2 k T} \\
& +O\left(\left(\frac{\hbar \omega}{2 k T}\right)^{2} \frac{1}{2 \Xi(\omega, T)}\right)
\end{aligned}
$$

where the function $3 \Xi(\omega, T)=2 \omega \tau\left(E^{*}\right)+E^{*} / k T$, taken together with eqn (5), depends on the classical parameters only. These functions were calculated recently for different types of the interaction potentials. ${ }^{5,6}$ For an exponential repulsive potential, $\Xi(\omega, T)$ follows as $\left(T_{\mathrm{LT}} / T\right)^{1 / 3}$ with $T_{\mathrm{LT}}=\pi^{2} \omega^{2} \mu a^{2} / 2 k$.

With decreasing $T$, all terms in eqn (25) (except the constant term) increase but the higher-order terms increase progressively faster. Ultimately, the expansion in eqn (25) breaks down, and the general expression of eqn (15) and (16) should be used instead. However, if the last term in eqn (25) remains small, i.e. $(\hbar \omega)^{2} /(2 k T)^{2} \ll 2 \Xi(\omega, T)$, the curvature of the generalized plot of $\ln \left\{\exp (\hbar \omega / 2 k T)\left\langle P_{10}\right\rangle\right\} \quad$ vs $T^{-1 / 3}$ can be unambiguously attributed to the deviation of the interaction potential from a purely exponential repulsive form.
In order to clearly see the effects of higher-order quantum correction in the temperature dependence of $\left\langle P_{10}^{\mathrm{QC}}\right\rangle$ we consider an interaction of the exponential form.

For this case, the temperature dependence of $\left\langle P_{10}^{\mathrm{QC}}\right\rangle$ enters through the reduced temperature $\theta=T / T_{\mathrm{LT}}$, while quantum effects are expressed through a temperature-independent parameter $\theta_{\text {vib }}=\hbar \omega / k T_{\mathrm{LT}}$. Referring to eqn (12), one may rewrite eqn (15) in the form

$$
\left\langle P_{10}^{\mathrm{QC}}\left(\theta, \theta_{\text {vib }}\right)\right\rangle=\mathrm{A} \int_{0}^{\infty} \exp \left[\frac{4 \theta^{1 / 2}}{\theta_{\text {vib }}}\left(\sqrt{\varepsilon}-\sqrt{\varepsilon+\theta_{\text {vib }} / \theta}\right)-\varepsilon\right] \mathrm{d} \varepsilon
$$

where the integration variable is $\varepsilon=E_{i} / k T$ and $\mathrm{A}$ is a temperature-independent factor. Eqn (26) is written in full integral form rather than in the SD approximation, since the latter requires the solution of a transcendental equation (see eqn (17)) that introduces unnecessary technical complications. The CCT (corrected CT) and CT (original Landau-Teller formulation) counterparts of eqn (26) are

$$
\begin{aligned}
\left\langle P_{10}^{\mathrm{CCT}}\left(\theta, \theta_{\mathrm{vib}}\right)\right\rangle & =\mathrm{A} \exp \left(\theta_{\mathrm{vib}} / 2 \theta\right) \int_{0}^{\infty} \exp \left(-2 \theta^{-1 / 2} \varepsilon^{-1 / 2}-\varepsilon\right) \mathrm{d} \varepsilon \\
& \rightarrow \mathrm{A} \sqrt{\frac{4 \pi}{3}} \theta^{-1 / 6} \exp \left(-3 \theta^{-1 / 3}+\theta_{\mathrm{vib}} / 2 \theta\right) \\
\left\langle P_{10}^{\mathrm{CT}}(\theta)\right\rangle & =\mathrm{A} \int_{0}^{\infty} \exp \left(-2 \theta^{-1 / 2} \varepsilon^{-1 / 2}-\varepsilon\right) \mathrm{d} \varepsilon \\
& \rightarrow \mathrm{A} \sqrt{\frac{4 \pi}{3}} \theta^{-1 / 6} \exp \left(-3 \theta^{-1 / 3}\right)
\end{aligned}
$$

where the arrow means "in the SD approximation". The additional preexponential factors that appear in the SD approximation in eqn (27) and (28) are only included for consistency with the integral representation, they do not affect the main temperature dependence of transition probabilities in any visible way.

Fig. 1 shows the logarithm (base 10) of the derived transition probabilities $v s \theta^{-1 / 3}=\left(T_{\mathrm{LT}} / T\right)^{1 / 3}$ (the LT representation) for a value of $\theta_{\mathrm{vib}}=\hbar \omega / k T_{\mathrm{LT}}=0.012$ which is appropriate for $\mathrm{N}_{2}$ or $\mathrm{CO}$ relaxation in He. The original LT transition probability, $\left\langle P_{10}^{\mathrm{CT}}(\theta)\right\rangle$, eqn $(28)$, is presented by the straight line of curve 1 , the corrected LT transition probability, $\left\langle P_{10}^{\mathrm{CCT}}\left(\theta, \theta_{\mathrm{vib}}\right)\right\rangle$, eqn (27), by curve 2 and the QC Landau probability, $\left\langle P_{10}^{\mathrm{QC}}\left(\theta, \theta_{\mathrm{vib}}\right)\right\rangle$, eqn (26), by curve 3 . The range of $\theta$ is chosen from the condition that the transition probabilities vary over several orders of magnitude. The first quantum correction shows itself as a spacing between curves 2 and 1 , and the condition of applicability of the first correction is formulated as a small spacing between curves 2 and 3 . The importance of the first correction is illustrated by the comparison of $\left\langle P_{10}^{\mathrm{QC}}\left(\theta, \theta_{\mathrm{vib}}\right)\right\rangle,\left\langle P_{10}^{\mathrm{CCT}}\left(\theta, \theta_{\mathrm{vib}}\right)\right\rangle$, and $\left\langle P_{10}^{\mathrm{CT}}(\theta)\right\rangle$. When the first quantum correction increases the LT transition probability $\left\langle P_{10}^{\mathrm{CT}}(\theta)\right\rangle$ by one order of magnitude, changing it to $\left\langle P_{10}^{\mathrm{CCT}}\left(\theta, \theta_{\mathrm{vib}}\right)\right\rangle$ and bringing it close to $\left\langle P_{10}^{\mathrm{QC}}\left(\theta, \theta_{\mathrm{vib}}\right)\right\rangle$ (this happens at about $\left.\theta^{-1 / 3}=8\right)$, the higher-order corrections became visible in the plot. With a decrease in temperature, the 


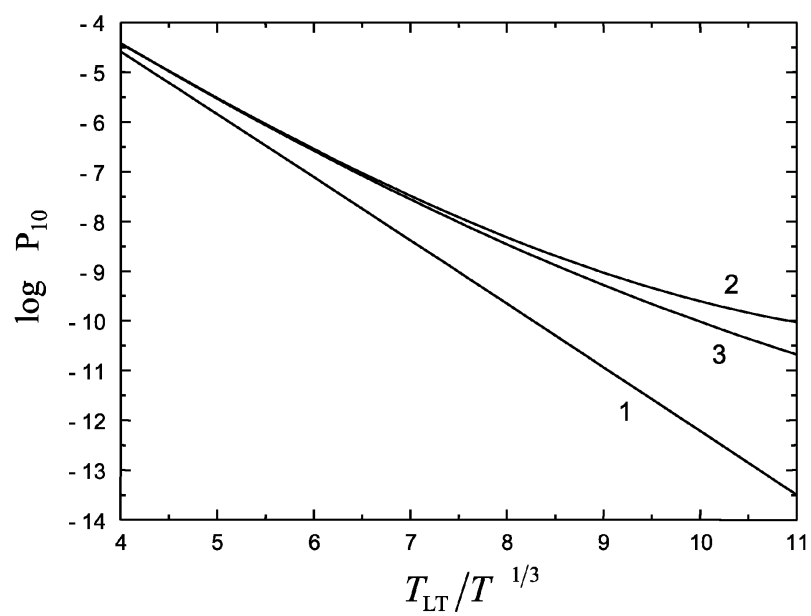

Fig. 1 LT plots for an exponential interaction potential: logarithms of mean thermal transition probabilities $\left\langle P_{10}\right\rangle$ vs $\left(T_{\mathrm{LT}} / T\right)^{1 / 3}$ for an arbitrarily fixed preexponential factor $\mathrm{A}=1$. The straight line 1 corresponds to the original LT probability (common trajectory approximation), line 2 to the corrected LT probability, and curve 3 to the Landau probability (QC approximation). Curves 2 and 3 are drawn for $\hbar \omega / k T_{\mathrm{LT}}=0.012$, see text.

importance of higher-order corrections rapidly grows. For instance, for $\mathrm{N}_{2}+\mathrm{He}$ collisions, this behavior does not permit safe extrapolations of the probability in the form $\left\langle P_{10}^{\mathrm{EFC}}(\theta\right.$, $\left.\left.\theta_{\text {vib }}\right)\right\rangle$ from higher temperatures down to room temperature. A comparison of CT, CCT and QC approximations with accurate quantum calculations and experimental data for specific systems would require using a realistic atom-molecule interaction potential and accounting for the three-dimensional character of the collision. This is done for the $\mathrm{N}_{2}-\mathrm{He}$ system in ref. 24.

An example for a complete failure of the corrected LT expression is provided by vibrational predissociation, where vibrational down-transitions in the oscillator $\mathrm{BC}$ induce the cleavage of a weak van der Waals bond A-BC. In this case $P^{\mathrm{QC}}\left(E_{i}, E_{f}\right)$ refers to the probability of dissociation per single oscillation of the A-BC bond at the bound-state energy $E_{i}$. If the energy transfer $\Delta E$ by far exceeds the dissociation energy $D$, the value of the integral in eqn (9) is dominated by the repulsive region of interaction which can be roughly taken as being exponential. Employing again $\tau(E)=\pi a / \sqrt{2 E / \mu}$ we then get the approximate relation

$$
\left.\frac{2}{\hbar} \int_{E_{i}}^{E_{i}+\Delta E} \tau(E) \mathrm{d} E\right|_{\left|E_{i}\right|<\Delta E} \approx \frac{2 \pi a}{\hbar} \sqrt{2 \mu \Delta E}
$$

This estimate relates the predissociation rate with the energy release through

$$
P(\Delta E) \propto \exp \left(-\frac{2 \pi a}{\hbar} \sqrt{2 \mu \Delta E}\right)
$$

and represents the so-called "momentum gap law": the rate decreases exponentially with an increase of the relative momentum of the receding fragments.

Yet another example for the application of the Landau expression in the form of eqn (10) is found in the similarity and difference of quantum and classical treatments of predissociation dynamics, see ref. 25.

\section{Conclusion}

The article by Landau and Teller from the year $1936^{1}$ had enormous impact on the understanding of energy transfer in molecular collisions, both on the level of state-selected rate coefficients and of macroscopic energy relaxation kinetics. The authors realized that their approximate calculations of stateselected rate coefficients should not disobey detailed balance, since the latter is responsible for attaining the correct equilibrium of the relaxing system. Therefore, they forced their approximate state-selected rate coefficients to comply with the detailed balance relation. They suspected that by doing this, they might have affected the values of the rate coefficients, and, indeed, they did affect the values. In the present treatment it became possible to correct this shortcoming by rederiving the LT results from a more general approach formulated by Landau in $1932^{16}$ and by recasting the rate coefficients in a form which allows one to express the Landau quasi-classical exponent through the LT classical collision time. ${ }^{19}$

\section{Acknowledgements}

Fruitful discussions with G. Käb and A. Neufeld on the quantum-classical description of system-bath couplings are acknowledged.

\section{References}

1 L. Landau and E. Teller, Phys. Z. Sowjetunion, 1936, 10, 34.

2 E. E. Nikitin, in Gas Phase Chemical Reaction System, ed. J. Wolfrum, H.-R. Volpp, R. Rannacher and J. Warnatz, Springer, Berlin-Heidelberg, 1996, p. 231.

3 C. T. Wickham-Jones, H. T. Williams and C. J. S. M. Simpson, J. Chem. Phys., 1987, 87, 5294.

4 E. E. Nikitin, Theory of Elementary Atomic and Molecular Processes in Gases, Clarendon Press, Oxford, 1974.

5 D. Schwarzer and M. Teubner, J. Chem. Phys., 2002, 116, 5680.

6 M. Teubner, Phys. Rev. E, 2002, 65, 031204.

7 D. W. Oxtoby, Adv. Chem. Phys., 1981, 47, 487.

8 S. A. Egorov and J. L. Skinner, Chem. Phys. Lett., 1998, 283, 469.

9 S. A. Egorov, E. Rabinim and B. J. Berne, J. Phys. Chem. B, 1999, 103, 10978.

10 G. Käb, Phys. Rev. E, 2002, 66, 046117.

11 G. Käb, J. Phys. Chem. A, 2004, 108, 8866.

12 A. A. Neufeld, J. Chem. Phys., 2003, 119, 2488.

13 A. A. Neufeld, D. Schwarzer, J. Schröder and J. Troe, J. Chem. Phys., 2003, 119, 2502

14 Q. Shi and E. Geva, J. Phys. Chem. A, 2003, 107, 9059.

15 Q. Shi and E. Geva, J. Phys. Chem. A, 2003, 107, 9070.

16 L. Landau, Phys. Z. Sowjetunion, 1932, 2, 46.

17 E. W. Montroll and K. E. Shuler, J. Chem. Phys., 1957, 26, 454.

18 L. D. Landau and E. M. Lifshitz, Quantum Mechanics (Nonrelativistic Theory), Pergamon Press, Oxford, 1977.

19 E. E. Nikitin and L. Pitaevski, Phys. Rev. A, 1994, 49, 695.

20 J. M. Jackson and N. F. Mott, Proc. R. Soc. London, Ser. A, 1932, 137, 703.

21 E. E. Nikitin, Opt. Spektrosk., 1959, 6, 93.

22 P. Schofield, Phys. Rev. Lett., 1960, 4, 239.

23 A. Miklavc, J. Chem. Phys., 1983, 78, 4502.

24 E. I. Dashevskaya, I. Litvin, E. E. Nikitin and J. Troe, J. Chem. Phys., submitted.

25 E. E. Nikitin and J. Troe, in Theory of Chemical Reaction Dynamics, ed. A. Lagana and G. Lendvay, Kluwer, Dordrecht, The Netherlands, 2004, p. 381. 